\title{
A novel role for TNFAIP2: its correlation with invasion and metastasis in nasopharyngeal carcinoma
}

\author{
Lih-Chyang Chen ${ }^{1}$, Chia-Chun Chen ${ }^{2}$, Ying Liang ${ }^{1}$, Ngan-Ming Tsang ${ }^{3}$, Yu-Sun Chang ${ }^{1,2}$ \\ and Chuen Hsueh ${ }^{4}$ \\ ${ }^{1}$ Chang Gung Molecular Medicine Research Center, Chang Gung University, Taoyuan, Taiwan; \\ ${ }^{2}$ Graduate Institute of Basic Medical Sciences, Chang Gung University, Taoyuan, Taiwan; \\ ${ }^{3}$ Department of Radiation Oncology, Chang Gung Memorial Hospital at Lin-Kou, Taoyuan, Taiwan and \\ ${ }^{4}$ Department of Pathology, Chang Gung Memorial Hospital at Lin-Kou, Taoyuan, Taiwan
}

\begin{abstract}
Tumor necrosis factor alpha (TNF $\alpha$ ) is an inflammatory cytokine that is present in the microenvironment of many tumors and is known to promote tumor progression. To examine how TNF $\alpha$ modulates the progression and metastasis of nasopharyngeal carcinoma, we used Affymetrix chips to identify TNF $\alpha$-inducible genes that are dysregulated in this tumor. Elevated expression of TNFAIP2, which encodes TNF $\alpha$-inducible protein 2 and not previously known to be associated with cancer, was found and confirmed by quantitative RT-PCR of TNFAIP2 expression in nasopharyngeal carcinoma and adjacent normal tissues. Immunohistochemical analysis showed that the TNFAIP2 protein was highly expressed in tumor cells. Analysis of $\mathbf{9 5}$ nasopharyngeal carcinoma biopsy specimens revealed that high TNFAIP2 expression was significantly correlated with highlevel intratumoral microvessel density $(P=0.005)$ and low distant metastasis-free survival $(P=0.001)$. A multivariate analysis further confirmed that TNFAIP2 was an independent prognostic factor for nasopharyngeal carcinoma $(P=0.002)$. In vitro, TNF $\alpha$ treatment of nasopharyngeal carcinoma HK1 cells was found to induce TNFAIP2 expression, and siRNA-based knockdown of TNFAIP2 dramatically reduced the migration and invasion of nasopharyngeal carcinoma HK1 cells. These results collectively suggest for the first time that TNFAIP2 is a cell migration-promoting protein and its expression predicts distant metastasis. Our data suggest that TNFAIP2 may serve as an independent prognostic indicator for nasopharyngeal carcinoma.

Modern Pathology (2011) 24, 175-184; doi:10.1038/modpathol.2010.193; published online 5 November 2010
\end{abstract}

Keywords: metastasis; migration; nasopharyngeal carcinoma; TNFAIP2

Tumor necrosis factor alpha (TNF $\alpha)$, a major inflammatory cytokine in the tumor microenvironment, is produced by both malignant and non-cancerous cells, ${ }^{1,2}$ and has been shown to mediate cancerrelated inflammation and promote tumor progression by enhancing angiogenesis and metastasis. ${ }^{3}$ Increased $\mathrm{TNF} \alpha$ levels in the tumor microenvironment and plasma have been associated with poor prognosis in various cancers, including prostate cancer, ${ }^{4}$ breast cancer, ${ }^{5}$ and nasopharyngeal carcinoma. $^{6,7}$ However, the mechanisms through

Correspondence: Dr C Hsueh, MD, Department of Pathology, Chang Gung Memorial Hospital at Lin-Kou, 5, Fu-Hsing Street, Kwei-Shan, Taoyuan 333, Taiwan.

E-mail: ch9211@adm.cgmh.org.tw

Received 8 June 2010; revised 25 August 2010; accepted 30 August 2010; published online 5 November 2010 which $\mathrm{TNF} \alpha$ increases tumor progression have not yet been fully defined.

Nasopharyngeal carcinoma is a highly metastatic head and neck cancer that is relatively rare among Caucasians, but is common in southeastern China and in Taiwan. ${ }^{8}$ In Hong Kong, the incidence of nasopharyngeal carcinoma is as high as $20-30$ per 100000 in men and 15-20 per 100000 in women. ${ }^{8}$ According to data from the Cancer Registry of Taiwan, nasopharyngeal carcinoma is the ninth most common cancer, with an estimated incidence of $\sim 6$ cases per 100000 in 2002. Under the current treatment regimens, the survival rates among nasopharyngeal carcinoma patients are $\sim 92 \%$ at 1 year and $\sim 50 \%$ at 5 years, with $20-25 \%$ of patients eventually developing distant metastases. ${ }^{9,10}$ Nasopharyngeal carcinoma is generally sensitive to radiation therapy, but more advanced cases may require a combination of radiotherapy and 
chemotherapy. ${ }^{11}$ Thus, it is essential for us to develop more effective diagnostic and therapeutic strategies against nasopharyngeal carcinoma.

In an effort to clarify the underlying mechanisms of malignancy in nasopharyngeal carcinoma, our group previously used Affymetrix-chip-based microarray hybridization to identify various nasopharyngeal carcinoma metastasis-associated biomarkers. We analyzed the various $\mathrm{TNF} \alpha$-regulated genes that appeared to be upregulated in nasopharyngeal carcinoma tumor cells vs adjacent normal tissues, and found differential expression of various $\mathrm{TNF} \alpha$ target genes MMP9, ${ }^{12}$ MMP12 ${ }^{13}$ MMP14, ${ }^{14}$ BIRC5, ${ }^{15}$ CXCL13, ${ }^{16}{ }^{1 L} 12 B,{ }^{17} C$-FLIP, ${ }^{18}$ and thymidine phosphorylase. ${ }^{19-21}$ We further showed that the upregulation of thymidine phosphorylase in nasopharyngeal carcinoma tumor cells resulted at least in part from cytoplasmic heterogeneous nuclear ribonucleoprotein $\mathrm{K}$ (hnRNP K)-mediated stabilization of the thymidine phosphorylase mRNA. ${ }^{22}$ High levels of thymidine phosphorylase and cytoplasmic hnRNP K were significantly correlated with overall survival and distant metastasis. ${ }^{19}$ On the basis of this success, we have continued to search for other $\mathrm{TNF} \alpha$ regulated genes that could serve as potential biomarkers for nasopharyngeal carcinoma prognosis.

TNFAIP2 is also a TNF $\alpha$-regulated gene that is expressed in endothelial cells, ${ }^{23}$ peripheral blood monocytes, ${ }^{24}$ and mature sperm. ${ }^{24}$ Here we investigated TNFAIP2, which is found to be highly expressed at both the mRNA and protein levels in nasopharyngeal carcinoma tumor cells vs adjacent normal tissues. TNFAIP2 encodes a protein whose precise function is not known, but appears to be involved in endothelial capillary tube formation in vitro. ${ }^{23}$ Although TNFAIP2 has been classified as an angiogenic factor, ${ }^{23}$ its function and significance in tumor development have not previously been determined. Here, we show for the first time that increased expression of TNFAIP2 is significantly correlated with shorter distant metastasis-free survival in nasopharyngeal carcinoma patients, and that TNFAIP2 promotes the migration and invasion of nasopharyngeal carcinoma cells in vitro. These results suggest that TNFAIP2 may serve as a potential diagnostic and independent prognostic marker for nasopharyngeal carcinoma.

\section{Materials and methods}

\section{Patients, Clinical Staging, Treatment, and Assessment} of Clinical Outcome

The utilized retrospective cohort comprised 95 nasopharyngeal carcinoma patients who had been admitted to Chang Gung Memorial Hospital at Lin-Kou from 1990 to 2006. AJCC stage, T stage, and N stage were defined according to the 2002 cancer-staging system revised by the American Joint Committee on Cancer. Histological typing was performed according to the WHO classification criteria, as described previously. ${ }^{19}$ This study was reviewed and approved by the institutional review board and ethics committee of Chang Gung Memorial Hospital. Informed consent was obtained from all patients.

All enrolled patients had been treated with definitive radiotherapy (cumulative dose of external beam radiotherapy $>$ R64.8 Gy). Among them, 33 patients had received additional chemotherapy in the Department of Medical Oncology at Chang Gung Memorial Hospital. Patients who presented with distant metastatic disease at diagnosis (M1 stage) and those who had undergone previous treatment at another institute were excluded from this study. For all enrolled patients, pathology reports were obtained from the pathological database and medical records, and the relevant slides were reviewed to confirm the nasopharyngeal carcinoma diagnosis (reviews were performed by C Hsueh). Clinical data on stage, treatment, and follow-up were collected from hospital tumor registries and medical files. The primary end point was disease-free survival, which was calculated from the date of diagnosis to the date of death or last follow-up. Distant metastasis-free survival and local recurrence-free survival were also assessed. The time to local recurrence or distant metastasis was calculated by using the date on which the local recurrence or distant metastasis was detected as the end point. Patients who died without local recurrence or distant metastasis were omitted from our analyses of disease-free survival, local recurrence-free survival, and distant metastasis-free survival.

\section{Affymetrix Microarray Analysis}

RNA samples from nasopharyngeal carcinoma tissues were isolated using the TRIzol reagent (Invitrogen, Carlsbad, CA, USA). The biotinylated oligonucleotide was hybridized to the Human Genome U133 Plus 2.0 Array (Affymetrix, Santa Clara, CA, USA) by the National Yang-Ming University Genomics Center (Taiwan), following the manufacturer's instructions. The microarray data were analyzed using the GeneSpring version GX 7.31 software package (Silcogenetics, Redwood, CA, USA). The relative expression level of each gene was calculated as the ratio of the average hybridization intensity from nine individual nasopharyngeal carcinoma tissues $v s$ that from a pool of the corresponding adjacent non-tumor tissues.

\section{Immunohistochemical Staining Analysis}

Immunohistochemical analyses were performed using an automatic immunohistochemical staining device according to the manufacturer's instructions (Bond-max Automated Immunostainer; Vision BioSystems, Melbourne, Australia). Tissue sections were retrieved using Bond Epitope Retrieval Solution 1 (Vision BioSystems) and stained with antibodies against TNFAIP2 (mouse monoclonal 
antibody, 1:100 dilution; Santa Cruz Biotechnology, Santa Cruz, CA, USA) and CD34 (mouse monoclonal antibody, ready to use; DAKO, Glostrup, Denmark). A polymer detection system (Bond Polymer Refine; Vision BioSystems) was used to reduce nonspecific staining. Tissue sections were treated with liquid DAB reagent, using 3'-diaminobenzidine tetrahydrochloride as the chromogen and hematoxylin as the counterstaining reagent. The expression of TNFAIP2 was assessed by quantitative scoring of the staining intensity and the proportion of positively stained cells. Staining intensity was graded as 0,1 , 2 , or 3 to indicate undetectable, weak, moderate, and strong staining, respectively. These scores were multiplied by the percentage of cells that showed positive staining; the resulting scores, which were taken as reflecting protein expression, were used to classify the specimens/patients into two groups: 'high-level' TNFAIP2 expression (scores $>120$ ) and 'low-level' TNFAIP2 expression (scores $\leq 120$ ). For assessment of intratumoral microvessel density, we stained sections using an antibody against CD34 (an endothelial marker). Microvessel density was determined from $\times 100$ microscopic fields within the tumor areas containing the most microvessels. Vessels were counted from four different $\times 100$ fields, and the averages were calculated. Specimens averaging more than 14 microvessels per field were defined as 'high microvessel density', while those averaging 14 or fewer were defined as 'low microvessel density'. TNFAIP2-positive tumor cells and microvessel density in representative microscopic fields were scored independently by two experienced pathologists.

\section{Quantitative RT-PCR}

RNA samples from nasopharyngeal carcinoma tissues and HK1 cells were isolated using the TRIzol reagent (Invitrogen). Reverse transcription of RNA ( $1 \mu \mathrm{g})$ was performed using an ImProm-II kit (Promega, Madison, WI, USA) and oligo(dT) $)_{15}$ primers (Promega). The primers used to amplify the cDNA corresponding to TNFAIP2 were as follows: forward primer, $5^{\prime}$-CCTGCTCTCCCTACGC-3'; and reverse primer, $5^{\prime}$-CGTCCAAGATGCTCCG-3'. The primers used to amplify the GAPDH cDNA were as described previously. ${ }^{22}$ Quantitative RT-PCR was performed on a Light-Cycler (Roche Diagnostics, Mannheim, Germany), according to the manufacturer's instructions, using the FastStart DNA Master SYBR Green I reagent (Roche Diagnostics). The gene expression results were normalized with regard to the expression of GADPH.

\section{Cell Culture}

HK1 cells (a nasopharyngeal carcinoma cell line) were maintained in RPMI 1640 medium supplemented with $10 \%$ fetal bovine serum, $100 \mathrm{U} / \mathrm{ml}$ penicillin, and $100 \mu \mathrm{g} / \mathrm{ml}$ streptomycin at $37^{\circ} \mathrm{C}$ under $5 \% \mathrm{CO}_{2}$.

\section{Western Blotting}

Whole-cell lysates were prepared by suspending cells in NP40 lysis buffer (50 mM Tris-HCl, $\mathrm{pH} 7.5,150 \mathrm{mM}$ $\mathrm{NaCl}, 1 \%$ Igepal CA-630, $1 \mu \mathrm{g} / \mathrm{ml}$ leupeptin, $2 \mu \mathrm{g} / \mathrm{ml}$ aprotinin, $1 \mu \mathrm{g} / \mathrm{ml}$ pepstatin, and $1 \mathrm{mM}$ phenylmethylsulfonyl fluoride). The samples were incubated on ice for $30 \mathrm{~min}$, the resulting lysates were centrifuged at $12000 \mathrm{~g}$ at $4^{\circ} \mathrm{C}$ for $10 \mathrm{~min}$, and the supernatants were retained. The protein concentrations in the clarified lysates were determined using the Bradford reagent. Equal amounts of protein $(30 \mu \mathrm{g})$ were resolved by electrophoresis on 10\% SDSpolyacrylamide gels, and transferred to nitrocellulose membranes. The membranes were blocked with $5 \%$ non-fat dry milk in TBS-Tween 20 , and then incubated at room temperature for $2 \mathrm{~h}$ with primary anti-TNFAIP2 and anti-tubulin antibodies (Santa Cruz Biotechnology). The membranes were subsequently incubated with secondary antibodies coupled to horseradish peroxidase and the results were visualized using ECL detection reagents (Amersham Pharmacia Biotech, Arlington Heights, IL, USA).

\section{RNA Interference}

HK1 cells $\left(4 \times 10^{5}\right)$ were plated on six-well plates and transfected using $50 \mathrm{nmol} / \mathrm{l}$ dsRNA duplexes and $50 \mu \mathrm{g}$ dsRNA transfection reagents (TransITTKO; Mirus Bio, Madison, WI, USA), according to the manufacturer's protocol. SMARTpool reagents containing a mixture of four 21-bp RNA TNFAIP2targeting duplexes were purchased from Dharmacon (Lafayette, CO, USA), whereas the negative control siRNA were synthesized by Research Biolabs (Ayer Rajah Industrial Estate, Singapore). The TNFAIP2targeting oligonucleotide sequences were as follows: 5'-UAUAAAGGUUGGUUAGCUU-3'; 5'-CGAAUU ACAGGGCCAAUGU-3'; 5'-GAGCAGAAUUGGCAG GUAC-3'; and 5'-GGGAACCUAUCCAACAGUG-3'.

\section{Cell Proliferation Assay}

Cells were subjected to dsRNA transfection with control or TNFAIP2-targeting siRNAs, and total cell numbers were counted on days 1,2 , and 3 after transfection. Duplicate experiments were performed at least three independent times.

\section{ELISA Assay}

Cells were subjected to dsRNA transfection. At $48 \mathrm{~h}$ post-transfection, the transfection medium was replaced with complete medium. After $24 \mathrm{~h}$, the conditioned media were collected and centrifuged at 1500 r.p.m. for $5 \mathrm{~min}$. The resulting pellets were discarded and the supernatants were tested for vascular endothelial growth factor (VEGF) expression using the Quantikine Human VEGF 
Immunoassay ( $\mathrm{R}$ \& D Systems, Minneapolis, MN, USA), according to the manufacturer's instructions.

\section{Cell Migration and Invasion Assays}

The migration and invasion of HK1 cells were evaluated using a chemotaxis chamber (Corning, Corning, NY, USA) and a Cell Invasion Assay Kit (Chemicon, Temecula, CA, USA), respectively. HK1 cells that had been transfected with the dsRNA were washed twice with serum-free medium, resuspended in $100 \mu \mathrm{l}$ of serum-free medium, and added to the upper chamber of the apparatus. The lower chamber contained $10 \%$ FBS medium. For the migration and invasion assays, the cells were incubated for 24 and $72 \mathrm{~h}$, respectively. The migrated and invasive cells were fixed and stained for $15 \mathrm{~min}$ with $0.25 \%$ crystal violet, $10 \%$ formaldehyde, and $80 \%$ methanol, and then the filters were washed five times with $\mathrm{ddH}_{2} \mathrm{O}$ for the removal of non-adherent cells. Ten random fields $(\times 100$ magnification) were captured for each membrane, and the migrated or invasive cells were counted.

\section{Statistical Analysis}

All statistical analyses were performed using the SPSS 13.0 statistical software package (SPSS, Chicago, IL, USA). The relationships between TNFAIP2 expression and the microvessel density and various clinicopathological characteristics were evaluated using the Pearson $\chi^{2}$ test. Survival curves were plotted using the Kaplan-Meier method and compared using the log-rank test. The Cox proportional hazards model was applied for multivariate analysis to determine the independence of each prognostic factor. The cutoff values used to define high TNFAIP2 and microvessel density were based on the percentages of TNFAIP2 staining in tumor cells and average microvessel numbers, as determined from receiver operating characteristic curve analysis. The in vitro data were analyzed with the twotailed paired Student's $t$-test. The data shown represent the mean \pm s.d. or mean \pm s.e.m., as indicated.

\section{Results}

\section{Affymetrix Analysis of TNF $\alpha$-Inducible Genes in Nasopharyngeal Carcinoma}

$\mathrm{TNF} \alpha$ mediates cancer-related inflammation, and may promote tumor progression by increasing angiogenesis and metastasis. ${ }^{1-3,25}$ In an effort to identify genes that may be involved in $\mathrm{TNF} \alpha$ responsive malignant progression, we used the Affymetrix HG U133 Plus 2.0 chip to perform systematic microarray expression profiling on nine

Table 1 Overexpression of TNF $\alpha$-inducible genes in nasopharyngeal carcinoma

\begin{tabular}{rrr}
\hline Probe ID Gene symbol Gene full name & $\begin{array}{l}\text { Fold change } \\
\text { (nasopharyngeal } \\
\text { carcinoma/normal) }\end{array}$ \\
\end{tabular}

\begin{tabular}{lll}
\hline 207901_at & IL12B & Interleukin 12B \\
203936_s_at & MMP9 & Matrix metallopeptidase 9 \\
204933_s_at & TNFSF11 & Tumor necrosis factor receptor superfamily, member 11b \\
204580_at & MMP12 & Matrix metallopeptidase 12 \\
205242_at & CXCL13 & Chemokine (C-X-C motif) ligand 13 \\
202094_at & BIRC5 & Baculoviral IAP repeat-containing 5 \\
202509_s_at & TNFAIP2 & Tumor necrosis factor alpha-induced protein 2 \\
1552798_a_at & TLR4 & Toll-like receptor 4 \\
202628_s_at & SERPINE1 & $\begin{array}{l}\text { Serpin peptidase inhibitor, clade E (nexin, plasminogen activator } \\
\text { inhibitor type 1), member 1 }\end{array}$ \\
217279_x_at & MMP14 & Matrix metallopeptidase 14
\end{tabular}

67.9

40.5

29.5

27.8

23.6

18.2

17.66

17.48

17.26

16.96

This list of probe IDs of Human Genome U133 Plus 2.0 Array, which correspond to the top 10 of increased TNF $\alpha$-inducible response genes among total 1289 genes with more than twofold changes and $P$-value $<0.001$ between the nasopharyngeal carcinoma $(n=9)$ and a pooled normal.

\footnotetext{
Figure 1 TNFAIP2 is induced by TNF $\alpha$ and correlates with microvessel density in nasopharyngeal carcinoma. (a) Induction of TNFAIP2 by TNF $\alpha$ in nasopharyngeal carcinoma cells. The levels of TNFAIP2 protein were determined by western blotting of HK1 cells incubated with TNF $\alpha$ for $48 \mathrm{~h}$. Tubulin was measured as a loading control. TNFAIP2 mRNA levels were measured by quantitative RT-PCR, and the results were normalized with regard to those of glyceraldehyde-3-phosphate dehydrogenase (GAPDH). Control values were set as 1.0. All data shown represent the mean \pm s.d. from at least three experiments; ${ }^{*} P<0.001$. (b) TNFAIP2 mRNA expression in nasopharyngeal carcinoma specimens. Quantitative RT-PCR was used to examine the relative mRNA expression levels in nine nasopharyngeal carcinoma tissues and pooled adjacent normal tissues, and the results were normalized with regard to GAPDH expression. All data are presented as the mean \pm s.e.m. $(\mathbf{c}-\mathbf{j})$ Consecutive nasopharyngeal carcinoma tissue sections were stained using anti-TNFAIP2 and anti-CD34 antibodies, and subjected to immunohistochemical analysis. Images were taken at $\times 100$ magnification $(\mathbf{c}, \mathbf{e}, \mathbf{g}$ and $\mathbf{i})$ and $\times 400$ magnification (d, $\mathbf{f}, \mathbf{h}$ and $\mathbf{j})$. Elevated TNFAIP2 expression was observed primarily in tumor cells, whereas no or weak expression was seen in normal epithelium. Representative images of tumor cells with high (c and d) and low (e and f) CD34 expression and with high (g and $\mathbf{h}$ ) and low (i and $\mathbf{j})$ microvessel density are shown. The sample shown contains normal epithelium (N) and stroma (S), and the tumor (T), as indicated. The boxed areas depict $\times 400$ magnification; bars $=100 \mu \mathrm{M}$.
} 
nasopharyngeal carcinoma tumor tissues and a pool of adjacent normal tissues. We found that 1289 genes were significantly increased (by twofold or more) in nasopharyngeal carcinoma tissues vs adjacent normal tissues (cutoff value, $P<0.001$ ). The 10 most highly induced genes (Table 1) were a

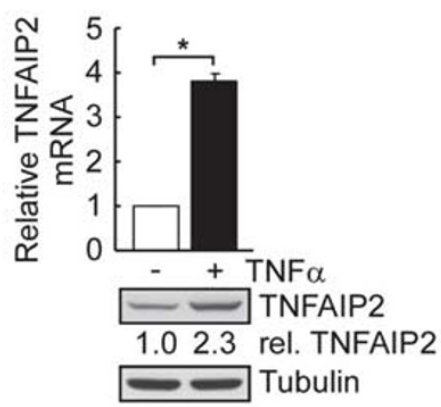

TNFAIP2

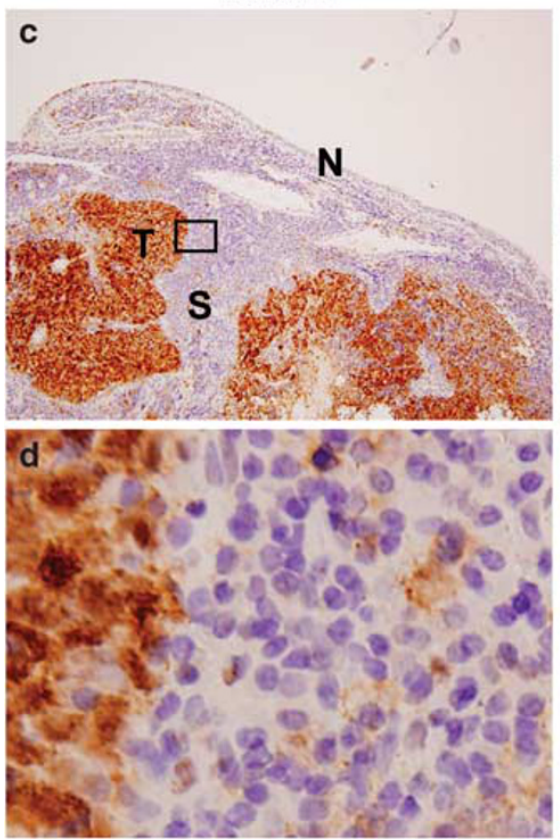

e
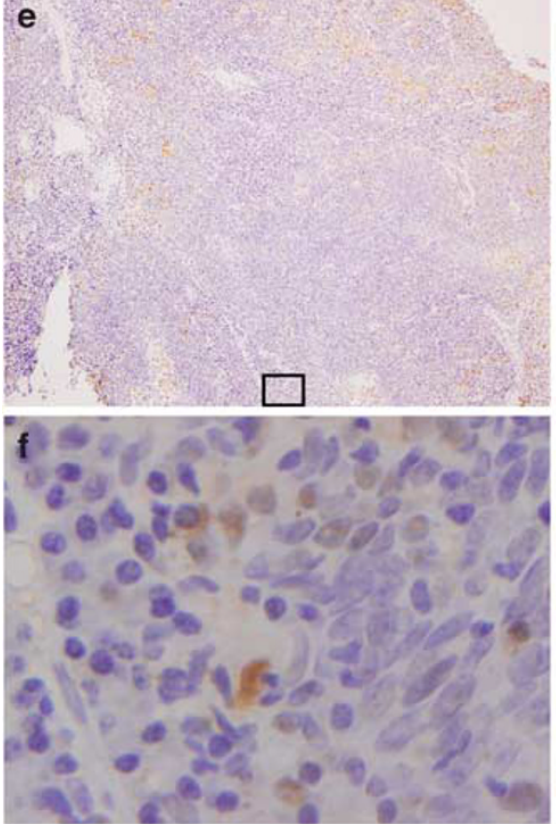

b

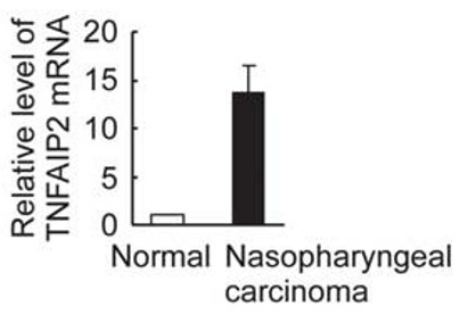

CD34
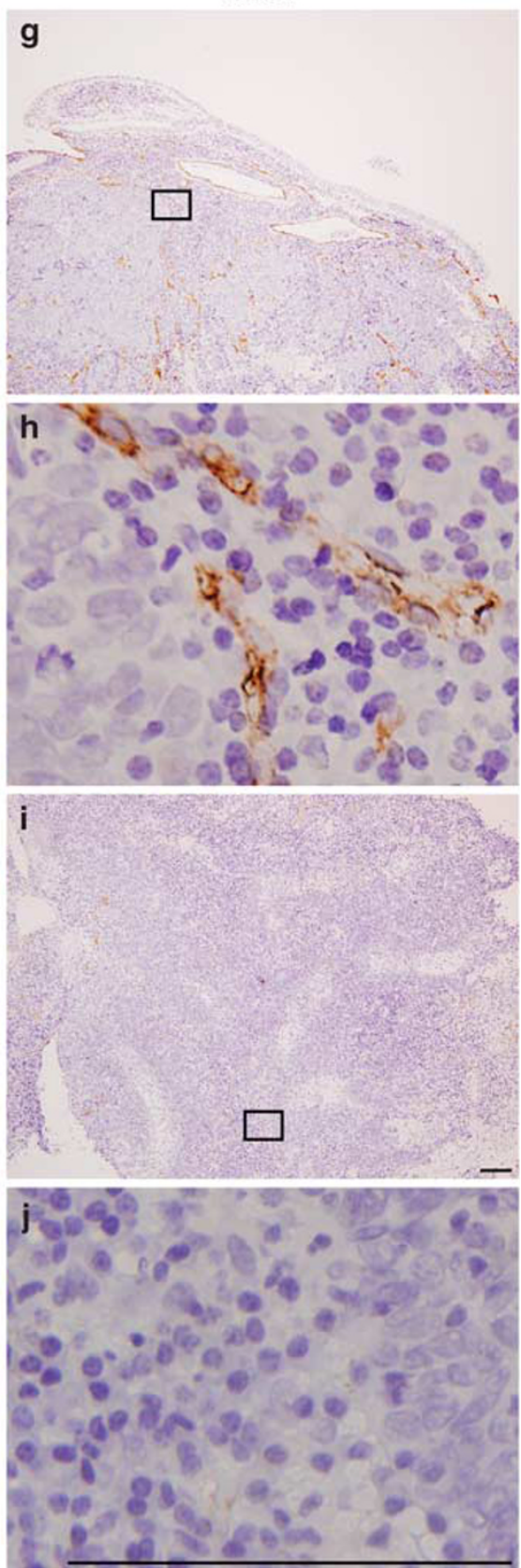
IL12B, ${ }^{17} \mathrm{MMP} 9,{ }^{12} \mathrm{TNFSF} 11,{ }^{26} \mathrm{MMP} 12,{ }^{13} \mathrm{CXCL13},{ }^{16}$ BIRC5, ${ }^{15}$ TNFAIP2, ${ }^{23}$ TLR $4,{ }^{27}$ SERPINE1, ${ }^{28}$ and $M M P-14 .{ }^{14}$ Consistent with our previous findings, $c$-FLIP ${ }^{29}$ and thymidine phosphorylase ${ }^{19}$ were also significantly increased, by 2.26 -fold (208485_x_at) and 3.46-fold (204858_s_at), respectively. From the highly upregulated genes, we chose to further explore TNFAIP2, as the expression, biological function, and potential pathological significance of this gene and its encoded protein had not been previously explored in the context of nasopharyngeal carcinoma or other cancers.

TNFAIP2 is Regulated by TNF $\alpha$, and Its Expression is Correlated with Microvessel Density in Nasopharyngeal Carcinoma

Although TNFAIP2 was originally identified as a gene that was rapidly induced by $\mathrm{TNF} \alpha$ treatment of endothelial cells, ${ }^{23}$ its regulation in nasopharyngeal carcinoma cells had not been studied previously. Accordingly, we first examined the effect of TNF $\alpha$ on the expression of TNFAIP2 in a nasopharyngeal carcinoma cell line (HK1 cells). As shown in Figure 1a, the mRNA and protein levels of TNFAIP2 were induced by 3.8- and 2.3-fold, respectively, in TNF $\alpha$-treated cells compared with control cells. This suggests that TNFAIP2 could be induced by $\mathrm{TNF} \alpha$ treatment of nasopharyngeal carcinoma cells.

To confirm the elevated expression of TNFAIP2 in nasopharyngeal carcinoma, we used quantitative RT-PCR to examine the levels of TNFAIP2 mRNA in RNA purified from nasopharyngeal carcinoma tissues. As shown in Figure 1b, the mRNA levels of TNFAIP2 were significantly elevated (by 13.7-fold) in nasopharyngeal carcinoma tissues $v s$ adjacent normal tissues. We further used immunohistochemical staining with an anti-TNFAIP2 antibody to examine TNFAIP2 protein expression in 95 nasopharyngeal carcinoma biopsy specimens. We detected high-level expression of TNFAIP2 in 39\% of the samples, and low-level expression in $61 \%$ (Figures 1c-f and Table 2). In contrast, TNFAIP2 expression was found to be undetectable or very weak in normal nasopharyngeal epithelial and stromal cells (Figures 1c and d).

As TNFAIP2 expression has been correlated with endothelial capillary tube formation in vitro, ${ }^{23}$ we further used immunohistochemical staining with an antibody against CD34 (an endothelial marker) to analyze the potential correlation of TNFAIP2 with intratumoral microvessel density. As shown in Figures 1g-j and Table 2, 60 (63\%) and 35 (37\%) of the samples showed high and low levels of microvessel density, respectively. Statistical analysis of the nasopharyngeal carcinoma expression data using the Pearson $\chi^{2}$ test showed that TNFAIP2 expression was positively correlated with microvessel density (Table 2; $P<0.005$ ).
Table 2 Relationship between TNFAIP2 and clinicopathological features

\begin{tabular}{|c|c|c|c|c|}
\hline \multirow[t]{2}{*}{ Characteristics } & \multirow[t]{2}{*}{ Total (n) } & \multicolumn{2}{|c|}{ TNFAIP2 } & \multirow[t]{2}{*}{$\mathrm{P}$-value } \\
\hline & & High & Low & \\
\hline Age & & & & 0.528 \\
\hline$>$ Median & 49 & 21 & 28 & \\
\hline$\leq$ Median & 46 & 16 & 30 & \\
\hline Gender & & & & 1.000 \\
\hline Male & 73 & 28 & 45 & \\
\hline Female & 22 & 9 & 13 & \\
\hline T stage & & & & $0.037^{\mathrm{a}}$ \\
\hline $1-2$ & 54 & 16 & 38 & \\
\hline $3-4$ & 41 & 21 & 20 & \\
\hline$N$ stage & & & & 1.000 \\
\hline $0-1$ & 51 & 20 & 31 & \\
\hline $2-3$ & 44 & 17 & 27 & \\
\hline AJCC stage & & & & 0.509 \\
\hline I-II & 33 & 11 & 22 & \\
\hline III-IV & 62 & 26 & 36 & \\
\hline Chemotherapy & & & & 0.662 \\
\hline No & 62 & 23 & 39 & \\
\hline Yes & 33 & 14 & 19 & \\
\hline Microvessel density & & & & $0.005^{\mathrm{a}}$ \\
\hline Low & 35 & 7 & 28 & \\
\hline High & 60 & 30 & 30 & \\
\hline
\end{tabular}

${ }^{\mathrm{a}}$ With statistical significance. Median age is 45 years.

\section{Patient Characteristics and TNFAIP2}

To evaluate the prognostic significance of TNFAIP2 under current therapeutic protocols, we studied the clinical outcomes among our cohort of 95 nasopharyngeal carcinoma patients. The patient characteristics and clinical features are summarized in Table 2. The median age at diagnosis was 45 years (range, 13.6-73.4 years), with a male-to-female ratio of $\sim 3: 1$. The clinicopathological features were comparable between the classified patient subgroups. No significant correlation was found between TNFAIP2 expression and age, gender, N stage, AJCC stage, or chemotherapy. Notably, however, TNFAIP2 expression was significantly associated with the extent of the primary tumor designated as $\mathrm{T}$ stage.

\section{Association of TNFAIP2 with Distant Metastasis-free Survival}

Kaplan-Meier survival analysis was used to compare disease-free survival, local recurrence-free survival, and distant metastasis-free survival between patients with high and low TNFAIP2 positivity. As shown in Figure 2, we found a significant difference in distant metastasis-free survival between patients with high and low TNFAIP2 $(P=0.001)$, whereas there was no statistical difference 

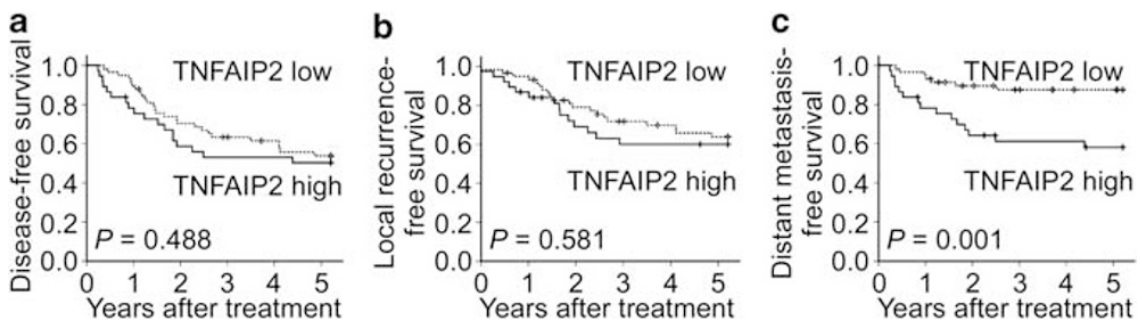

Figure 2 Kaplan-Meier survival analysis of nasopharyngeal carcinoma patient survival as a function of TNFAIP2 expression. (a) Diseasefree survival, (b) local recurrence-free survival and (c) distant metastasis-free survival. The patient subgroups were TNFAIP2 high $(n=37)$ and TNFAIP2 low $(n=58)$.

for disease-free survival or local recurrence-free survival $(P=0.488$ and 0.581 , respectively $)$. Among the 95 patients, 37 and 58 patients showed high and low TNFAIP2 expression, respectively, in their tumor cells. In the high expression group, 15 of 37 patients developed distant metastases after the initial radiotherapy $(40.5 \%)$, whereas this proportion was only seven of 58 in the low expression group $(12.1 \%)$. Thus, the expression level of TNFAIP2 was significantly correlated with distant metastasis-free survival $(P=0.001)$ in nasopharyngeal carcinoma patients. We next conducted a multivariate analysis of TNFAIP2 expression level with age, gender, T stage, $\mathrm{N}$ stage, chemotherapy, and microvessel density. Our results indicated that TNFAIP2 and N stage were independent prognostic predictors of poor distant metastasis-free survival $(P=0.002$ and 0.036 , respectively; Table 3$)$.

\section{Promotion of Cell Migration and Invasion by TNFAIP2}

As our results indicated that TNFAIP2 overexpression was correlated with intratumoral angiogenesis and distant metastasis in nasopharyngeal carcinoma patients, we next examined its biological functions in nasopharyngeal carcinoma HK1 cells, using RNA interference technology. First, we analyzed the effects of TNFAIP2 knockdown on the migration and invasion (two processes that are critical to distant metastasis) of HK1 cells. As shown in Figures $3 \mathrm{a}$ and b, TNFAIP2-knockdown cells showed significant reductions in cell migration and invasion, suggesting that TNFAIP2 may be involved in the migration and invasion of tumor cells. We then characterized the effect of TNFAIP2 knockdown on cell growth. As shown in Figure 3c, growth was not significantly different between TNFAIP2-knockdown and control siRNA-transfected HK1 cells, suggesting that TNFAIP2 does not affect tumor growth. Finally, we tested for a potential interaction between TNFAIP2 and VEGF, an important secreted angiogenesis-initiating factor that has also been correlated with microvessel density in nasopharyngeal carcinoma patients. ${ }^{30}$ As the protein sequence of TNFAIP2 (amino acids 91-651) is highly similar to that of SEC6 (based on a
Table 3 Cox multivariate regression analysis of the association between TNFAIP2 and distant metastasis-free survival of nasopharyngeal carcinoma patients

\begin{tabular}{|c|c|c|c|}
\hline \multirow[t]{2}{*}{ Characteristics } & \multicolumn{2}{|c|}{ Patients $(\mathrm{n}=95)$} & \multirow[t]{2}{*}{$\mathrm{P}$-value } \\
\hline & $\begin{array}{l}\text { Hazards } \\
\text { ratio }\end{array}$ & $\begin{array}{c}95 \% \text { Confidence } \\
\text { interval }\end{array}$ & \\
\hline Age & & & 0.361 \\
\hline$>$ Median & 1.00 & Reference & \\
\hline$\leq$ Median & 0.66 & $0.268-1.616$ & \\
\hline Gender & & & 0.688 \\
\hline Male & 1.00 & Reference & \\
\hline Female & 0.80 & $0.264-2.406$ & \\
\hline T stage & & & 0.440 \\
\hline $1-2$ & 1.00 & Reference & \\
\hline $3-4$ & 1.44 & $0.568-3.672$ & \\
\hline$N$ stage & & & 0.036 \\
\hline $0-1$ & 1.00 & Reference & \\
\hline $2-3$ & 2.74 & $1.066-7.067$ & \\
\hline Chemotherapy & & & 0.706 \\
\hline No & 1.00 & Reference & \\
\hline Yes & 1.20 & $0.466-3.088$ & \\
\hline Microvessel density & & & 0.055 \\
\hline Low & 1.00 & Reference & \\
\hline High & 0.38 & $0.144-1.021$ & \\
\hline TNFAIP2 & & & $0.002^{\mathrm{a}}$ \\
\hline Low & 1.00 & Reference & \\
\hline High & 5.32 & $1.821-15.536$ & \\
\hline
\end{tabular}

${ }^{\mathrm{a}}$ With statistical significance. Median age is 45 years.

publicly available website http://www.sanger.ac.uk/), an exocyst complex component involved in exocytosis $^{31}$ and secretion, ${ }^{32}$ we examined whether TNFAIP2 affected the secretion of VEGF by HK1 cells. As shown in Figure 3d, our results revealed that VEGF secretion by HK1 cells was not affected by TNFAIP2 knockdown. These findings indicate that the effect of TNFAIP2 on tumor cells may not be mediated via VEGF secretion, and that VEGF secretion in tumor cells does not appear to be regulated by TNFAIP2. 

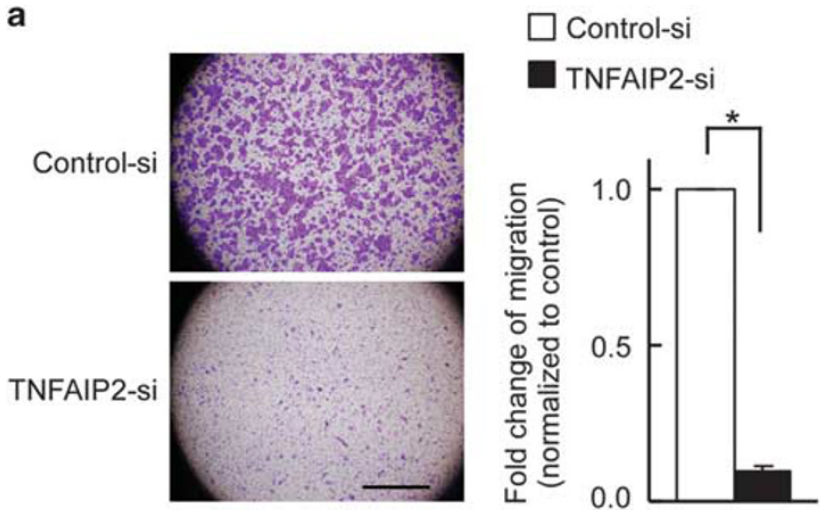

C
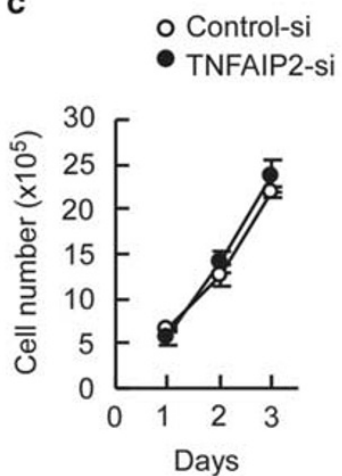

b

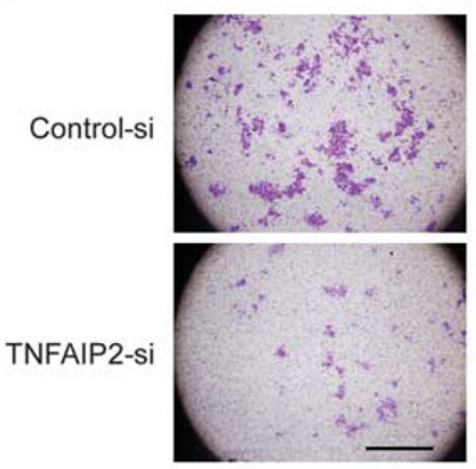

Control-si

TNFAIP2-si

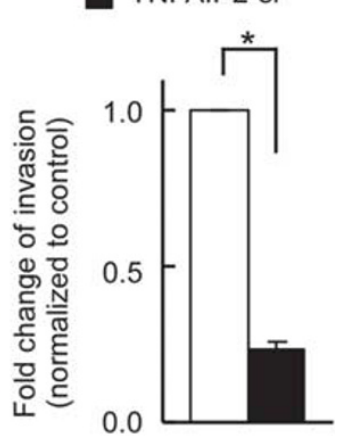

d
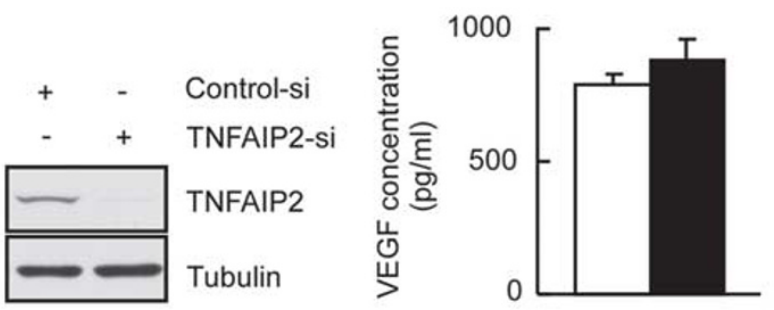

Figure 3 Promotion of cell migration and invasion by TNFAIP2. HK1 cells were transfected with TNFAIP2-targeting or control siRNA and then subjected to functional assays. (a) TNFAIP2 promotes cell migration. Trans-well migration assays were performed as described in the 'Materials and methods' section. Images were captured at $24 \mathrm{~h}$ under $\times 40$ magnification; bars $=1 \mathrm{mM}$. Cells were counted from 10 randomly picked fields and averages were calculated; results were obtained from three independent experiments. The relative foldchange in the number of migrated cells is shown, with the results from control cells given as 1.0; ${ }^{*} P<0.001$. (b) TNFAIP2 promotes cell invasion. Trans-well migration assays were performed as described in the 'Materials and methods' section. Images were captured at $72 \mathrm{~h}$ under $\times 40$ magnification; bars $=1 \mathrm{mM}$. The relative fold-change in the number of invasive cells was calculated as described in (a); ${ }^{*} P<0.001$. (c) TNFAIP2 knockdown does not appear to affect cell proliferation. Cell numbers were counted on several days following siRNA transfection. The representative protein levels of TNFAIP2 and tubulin (loading control) were determined by Western; the results from day 2 are shown and are similar to those obtained on days 1 and 3 (data not shown). (d) TNFAIP2 knockdown does not appear to affect VEGF secretion. HK1 cells were cultured for $24 \mathrm{~h}$ and the VEGF concentrations in the conditioned media were analyzed by ELISA; ${ }^{*} P<0.01$.

\section{Discussion}

This is the first report to show that TNFAIP2, which had previously been reported as a TNF $\alpha$-inducible protein, is highly expressed in nasopharyngeal carcinoma cells and is significantly correlated with intratumoral microvessel density and shorter distant metastasis-free survival in nasopharyngeal carcinoma patients. TNFAIP2 may therefore have the potential as an independent prognostic indicator for nasopharyngeal carcinoma. Importantly, we also herein find a novel role for TNFAIP2, by showing that it promotes the migration and invasion of nasopharyngeal carcinoma cells in vitro. This may explain the correlation of TNFAIP2 with the extent of tumor and distant metastasis-free survival in nasopharyngeal carcinoma patients.

In the tumor microenvironment, $\mathrm{TNF} \alpha$ mediates cancer-related inflammation, and may promote tumor progression by increasing angiogenesis and metastasis. ${ }^{1-3,25}$ The levels of $\mathrm{TNF} \alpha^{6-7}$ and its downstream genes $C$-FLIP $P^{18,29}$ and thymidine phosphorylase ${ }^{19-22}$ are known to be elevated in nasopharyngeal carcinoma tumor tissues. Here, we used Affymetrix chips to identify the $10 \mathrm{TNF} \alpha$-inducible genes showing the highest upregulation in nasopharyngeal carcinoma tissues vs normal adjacent tissues. Among them, we identified one gene that had not previously been examined in the context of cancer: TNFAIP2. The previous reports and our present findings collectively suggest that TNFAIP2 contributes to the tumor-promoting effects of $\mathrm{TNF} \alpha$. This hypothesis is supported by our present findings that: (1) TNFAIP2 was induced by TNF $\alpha$ treatment of nasopharyngeal carcinoma cells; (2) silencing of TNFAIP2 by siRNA-mediated knockdown significantly reduced the migration and invasion of HK1 cells (Figures 3a and b); and (3) TNFAIP2 expression levels were significantly correlated with shorter distant metastasis-free survival 
among 95 nasopharyngeal carcinoma patients. Taken together, these results suggest that the pro-tumor activity of $\mathrm{TNF} \alpha$ in nasopharyngeal carcinoma is mediated at least in part through TNFAIP2.

$\mathrm{TNF} \alpha$ has been shown to promote vascularization in rabbit cornea and chick chorioallantoic membrane,$^{33,34}$ and to induce the expression of various angiogenic factors, including VEGF. ${ }^{35-37}$ Furthermore, VEGF expression was found to be markedly increased and correlated with microvessel density in nasopharyngeal carcinoma patients. ${ }^{30}$ Similarly, we herein found that TNFAIP2 is a downstream target of $\mathrm{TNF} \alpha$ that acts as a potential angiogenic factor, as shown by the association of its expression with microvessel density in vivo.

The amino-acid sequence of TNFAIP2 is similar to that of SEC6, which is involved in exocytosis ${ }^{31}$ and secretion. ${ }^{32}$ Therefore, we hypothesized that TNFAIP2 could promote angiogenesis by upregulating VEGF secretion. However, we did not observe any decrease in VEGF secretion by TNFAIP2knockdown HK1 cells (Figure 3d), suggesting that TNFAIP2 is not involved in the regulation of VEGF secretion. As the regulatory network responsible for modulating angiogenesis is remarkably complex, further studies will be needed to clarify how TNFAIP2 affects angiogenesis in nasopharyngeal carcinoma.

TNFAIP2 has previously been shown to be regulated by interleukin- $1 \beta$ (IL-1 $\beta),{ }^{23}$ retinoic acid, ${ }^{38}$ erythroblastic leukemia viral oncogene homolog 2 (V-erb-b2), ${ }^{39}$ and dioxin. ${ }^{40}$ In the clinical setting, the levels of TNF $\alpha$ and IL- $1 \beta$ are known to be elevated in nasopharyngeal carcinoma. ${ }^{6,7,41}$ Thus, the dysregulation of TNFAIP2 expression in the nasopharyngeal carcinoma microenvironment may result from the combined effects of $\mathrm{TNF} \alpha$ and IL- $1 \beta$, which may promote tumor progression (eg, distant metastasis and angiogenesis) by inducing TNFAIP2.

In summary, we herein identify a novel role for TNFAIP2 by showing that it promotes the migration and invasion of nasopharyngeal carcinoma tumor cells. Moreover, we show that TNFAIP2 expression appears to be an independent prognostic marker for distant metastasis in nasopharyngeal carcinoma patients, and therefore might be a useful therapeutic indicator for these high-risk nasopharyngeal carcinoma patients to receive concurrent chemoradiotherapy.

\section{Acknowledgements}

We thank the Pathology Core of the Chang Gung Molecular Medicine Research Center for providing technical support. The study was supported by grants from the Ministry of Education (to Chang Gung University), National Science Council (NSC 98-3112-B-182-006 and 97-2320-B-182-001MY3) and Chang Gung Memorial Hospital (CMRPD160053), Taiwan.

\section{Disclosure/conflict of interest}

The authors declare no conflict of interest.

\section{References}

1 Sethi G, Sung B, Aggarwal BB. TNF: a master switch for inflammation to cancer. Front Biosci 2008;13:5094-5107.

2 Balkwill F. TNF-alpha in promotion and progression of cancer. Cancer Metast Rev 2006;25:409-416.

3 Balkwill F. Tumour necrosis factor and cancer. Nat Rev Cancer 2009;9:361-371.

4 Pfitzenmaier J, Vessella R, Higano CS, et al. Elevation of cytokine levels in cachectic patients with prostate carcinoma. Cancer 2003;97:1211-1216.

5 Bozcuk H, Uslu G, Samur M, et al. Tumour necrosis factor-alpha, interleukin-6, and fasting serum insulin correlate with clinical outcome in metastatic breast cancer patients treated with chemotherapy. Cytokine 2004;27:58-65.

6 Hsiao SH, Lee MS, Lin HY, et al. Clinical significance of measuring levels of tumor necrosis factor-alpha and soluble interleukin-2 receptor in nasopharyngeal carcinoma. Acta Otolaryngol 2009;129:1519-1523.

7 Yadav M. Endogenous tumor necrosis factor-alpha in patients with nasopharyngeal carcinoma. Southeast Asian J Trop Med Public Health 1991;22:123-126.

8 Wei WI, Sham JS. Nasopharyngeal carcinoma. Lancet 2005;365:2041-2054.

9 Lee AW, Poon YF, Foo W, et al. Retrospective analysis of 5037 patients with nasopharyngeal carcinoma treated during 1976-1985: overall survival and patterns of failure. Int J Radiat Oncol Biol Phys 1992;23: 261-270.

10 Tse KP, Tsang NM, Chen KD, et al. MCP-1 promoter polymorphism at 2518 is associated with metastasis of nasopharyngeal carcinoma after treatment. Clin Cancer Res 2007;13:6320-6326.

11 Guigay J. Advances in nasopharyngeal carcinoma. Curr Opin Oncol 2008;20:264-269.

12 Ries C, Kolb H, Petrides PE. Regulation of 92-kD gelatinase release in HL-60 leukemia cells: tumor necrosis factor-alpha as an autocrine stimulus for basal- and phorbol ester-induced secretion. Blood 1994;83:3638-3646.

13 Kerkela E, Ala-aho R, Klemi P, et al. Metalloelastase (MMP-12) expression by tumour cells in squamous cell carcinoma of the vulva correlates with invasiveness, while that by macrophages predicts better outcome. J Pathol 2002;198:258-269.

14 Ray BK, Shakya A, Turk JR, et al. Induction of the MMP-14 gene in macrophages of the atherosclerotic plaque: role of SAF-1 in the induction process. Circ Res 2004;95:1082-1090.

15 Takada Y, Khuri FR, Aggarwal BB. Protein farnesyltransferase inhibitor (SCH 66336) abolishes NF-kappaB activation induced by various carcinogens and inflammatory stimuli leading to suppression of NFkappaB-regulated gene expression and up-regulation of apoptosis. J Biol Chem 2004;279:26287-26299.

16 Suto H, Katakai T, Sugai M, et al. CXCL13 production by an established lymph node stromal cell line via lymphotoxin-beta receptor engagement involves the cooperation of multiple signaling pathways. Int immunol 2009;21:467-476. 
17 Kelleher P, Maroof A, Knight SC. Retrovirally induced switch from production of IL-12 to IL-4 in dendritic cells. Eur J Immunol 1999;29:2309-2318.

18 Spanaus KS, Schlapbach R, Fontana A. TNF-alpha and IFN-gamma render microglia sensitive to Fas ligandinduced apoptosis by induction of Fas expression and down-regulation of Bcl-2 and Bcl-xL. Eur J Immunol 1998;28:4398-4408.

19 Chen LC, Hsueh C, Tsang NM, et al. Heterogeneous ribonucleoprotein $\mathrm{k}$ and thymidine phosphorylase are independent prognostic and therapeutic markers for nasopharyngeal carcinoma. Clin Cancer Res 2008;14:3807-3813.

20 Chen CC, Chen LC, Liang Y, et al. Epstein-Barr virus latent membrane protein 1 induces the chemotherapeutic target, thymidine phosphorylase, via NF-kappaB and p38 MAPK pathways. Cell Signal 2010;22: 1132-1142.

21 Zhu GH, Lenzi M, Schwartz EL. The Sp1 transcription factor contributes to the tumor necrosis factor-induced expression of the angiogenic factor thymidine phosphorylase in human colon carcinoma cells. Oncogene 2002;21:8477-8485.

22 Chen LC, Liu HP, Li HP, et al. Thymidine phosphorylase mRNA stability and protein levels are increased through ERK-mediated cytoplasmic accumulation of hnRNP $\mathrm{K}$ in nasopharyngeal carcinoma cells. Oncogene 2009;28:1904-1915.

23 Sarma V, Wolf FW, Marks RM, et al. Cloning of a novel tumor necrosis factor-alpha-inducible primary response gene that is differentially expressed in development and capillary tube-like formation in vitro. J Immunol 1992;148:3302-3312.

24 Wolf FW, Sarma V, Seldin M, et al. B94, a primary response gene inducible by tumor necrosis factoralpha, is expressed in developing hematopoietic tissues and the sperm acrosome. J Biol Chem 1994;269: 3633-3640.

25 Colotta F, Allavena P, Sica A, et al. Cancer-related inflammation, the seventh hallmark of cancer: links to genetic instability. Carcinogenesis 2009;30:1073-1081.

26 Wei S, Kitaura H, Zhou P, et al. IL-1 mediates TNFinduced osteoclastogenesis. J Clin Invest 2005;115: 282-290.

27 Gutierrez-Canas I, Juarranz Y, Santiago B, et al. VIP down-regulates TLR4 expression and TLR4-mediated chemokine production in human rheumatoid synovial fibroblasts. Rheumatology (Oxford) 2006;45:527-532.

28 Mukai Y, Wang CY, Rikitake Y, et al. Phosphatidylinositol 3-kinase/protein kinase Akt negatively regulates plasminogen activator inhibitor type 1 expression in vascular endothelial cells. Am J Physiol Heart Circ Physiol 2007;292:H1937-H1942.

29 Chen LC, Chung IC, Hsueh C, et al. The antiapoptotic protein, FLIP, is regulated by heterogeneous nuclear ribonucleoprotein $\mathrm{K}$ and correlates with poor overall survival of nasopharyngeal carcinoma patients. Cell Death Differ 2010;17:1463-1473.

30 Guang-Wu H, Sunagawa M, Jie-En L, et al. The relationship between microvessel density, the expression of vascular endothelial growth factor (VEGF), and the extension of nasopharyngeal carcinoma. Laryngoscope 2000;110:2066-2069.

31 Hsu SC, TerBush D, Abraham M, et al. The exocyst complex in polarized exocytosis. Int Rev Cytol 2004;233:243-265.

32 Tsuboi $\mathrm{T}$, Ravier MA, Xie $\mathrm{H}$, et al. Mammalian exocyst complex is required for the docking step of insulin vesicle exocytosis. J Biol Chem 2005;280: 25565-25570.

33 Frater-Schroder M, Risau W, Hallmann R, et al. Tumor necrosis factor type alpha, a potent inhibitor of endothelial cell growth in vitro, is angiogenic in vivo. Proc Natl Acad Sci USA 1987;84:5277-5281.

34 Leibovich SJ, Polverini PJ, Shepard HM, et al. Macrophage-induced angiogenesis is mediated by tumour necrosis factor-alpha. Nature 1987;329:630-632.

35 Ryuto $\mathrm{M}$, Ono $\mathrm{M}$, Izumi $\mathrm{H}$, et al. Induction of vascular endothelial growth factor by tumor necrosis factor alpha in human glioma cells. Possible roles of SP-1. J Biol Chem 1996;271:28220-28228.

36 Yin Y, Wang S, Sun Y, et al. JNK/AP-1 pathway is involved in tumor necrosis factor-alpha induced expression of vascular endothelial growth factor in MCF7 cells. Biomed Pharmacother 2009;63:429-435.

37 Wang $X$, Lin Y. Tumor necrosis factor and cancer, buddies or foes? Acta Pharmacol Sin 2008;29:1275-1288.

38 Rusiniak ME, Yu M, Ross DT, et al. Identification of B94 (TNFAIP2) as a potential retinoic acid target gene in acute promyelocytic leukemia. Cancer Res 2000;60: 1824-1829.

39 Beckers J, Herrmann F, Rieger S, et al. Identification and validation of novel ERBB2 (HER2, NEU) targets including genes involved in angiogenesis. Int J Cancer 2005;114:590-597.

40 Rivera SP, Saarikoski ST, Sun W, et al. Identification of novel dioxin-responsive genes by representational difference analysis. Xenobiotica 2007;37:271-279.

41 Huang YT, Sheen TS, Chen CL, et al. Profile of cytokine expression in nasopharyngeal carcinomas: a distinct expression of interleukin 1 in tumor and CD4+ T cells. Cancer Res 1999;59:1599-1605. 